\title{
A model for retention and continuity of care and treatment for opioid dependent injection drug users in the Russian Federation
}

\author{
Elena Dmitrieva ${ }^{1}$, Sergei A. Frolov ${ }^{1}$, Thomas F. Kresina ${ }^{2^{*}}$, William Slater ${ }^{3}$ \\ ${ }^{1}$ Health and Development Foundation, Moscow, Russia \\ ${ }^{2}$ Division of Pharmacologic Therapies, Center for Substance Abuse Treatment, Substance Abuse and Mental Health Services Ad- \\ ministration, Rockville, USA; ${ }^{*}$ Corresponding Author: tkresina@samhsa.gov \\ ${ }^{3}$ Health Team, United States Agency for International Development, Moscow, Russia
}

Received 25 April 2012; revised 19 May 2012; accepted 29 May 2012

\begin{abstract}
Health and Development Foundation has worked collaboratively with the Government of the Russian Federation to develop and implement a model program of retention and continuity of care for opioid dependence and HIV infection. The model utilizes the Narcology Detoxification Service, State AIDS Centers, Non Government Organization (NGO) managed narcology treatment and rehabilitation centers, NGO outreach programs and the community. The model program was developed and implemented as a pilot demonstration project to provide essential health services to injection drug users and retain them in care. The interventions developed and implemented comprised HIV Testing and Counseling, HIVIAIDS Narcology Post-Graduate Curriculum, Peer Support Groups (Buffer groups), Narcological Follow-up Phone Monitoring, Women's Narcological Services and Short Messaging Services for Injection Drug Users. These services and interventions promoted the integration and utilization of HIVIAIDS health services and narcological services to form an evidencebased health service delivery model providing essential services to people who inject drugs and people living with HIVIAIDS in the Russian Federation.
\end{abstract}

Keywords: Opioid Dependence; Health Services; Retention in Care; Drug Treatment

\section{INTRODUCTION}

Exposure to addictive substances is widespread with the vulnerability to dependence being behaviorally complex as a function of biological, psychological and envi- ronmental interactions and influences [1]. In the Russian Federation, heroin is trafficked from Central Asia and readily available for use [2]. With continued opioid use, opioid dependence can occur rapidly and manifest as a chronic, relapsing brain disease. The clinical diagnosis of opioid dependence is not based on quantity of drug used, but on maladaptive patterns of drug use, as well as cognitive, behavioral, and physiological symptoms including any significant consequences related to drug use [3]. Treatment guidelines promote effective treatment programs that are multi-component and include pharmacotherapies with an array of services that address the cognitive, behavioral, physiological and social aspects of opioid dependence [4]. Opioid treatment programs also need to address the medical co-morbidities associated with opioid dependence and injection drug use, most significantly Human Immunodeficiency Virus (HIV). The World Health Organization (WHO) has developed a comprehensive set of interventions for HIV prevention, care and treatment for injection drug users [5]. They are: needle and syringe programs; drug dependence treatment; target information, education and communication for people who use drugs; HIV testing and counseling; HIV care and treatment; promoting and supporting safe and effective condom use; detection and management of sexually transmitted infections; prevention and treatment of viral hepatitis; tuberculosis prevention, diagnosis and treatment. Since 2005, Health and Development Foundation has worked collaboratively with the Government of the Russian Federation to develop and implement a model program of retention and continuity of care for individuals who are opioid dependent with HIV infection. This model program collaborative utilizes the Narcology Detoxification Service, State AIDS Centers, Non Government Organization (NGO) managed narcology treatment and rehabilitation centers, NGO outreach programs and the community to provide effective outreach to peo- 
ple who use drugs and promote their utilization of health care services, as well as, retention in care and treatment.

\section{OPIOID DEPENDENCE AND THE HIV EPIDEMIC IN THE RUSSIAN FEDERATION}

Because of the large amount of opioids trafficked from Central Asia through the Russian Federation, there is access to and availability of inexpensive heroin. Data from 2009, indicate that there are over 550,000 people in the Russian Federation who are officially registered as drug users [6] and 567,558 people living with HIV [7]. The world drug report estimates that there are approximately 1.6 million people in the Russian Federation who use opioids [2]. In some regions of the Russian Federation, it is estimated that the HIV prevalence in the opioid dependent populations can reach as high as $75 \%$ [2]. These official statistics for the Russian Federation indicate that from 1987 to 2008 , approximately $80 \%$ of all HIV infections were associated with injection drug use and opioid dependence [8]. Thus, the Russian Federation has a serious dual epidemic of opioid dependence and HIV infection. To address this dual epidemic, an integrated program of HIV prevention and care, in the context of the prevention and care of opioid dependence, was developed and implemented as a pilot demonstration project to provide essential health services to injection drug users and retain them in care.

\section{COMPONENT INTERVENTIONS OF THE MODEL PROGRAM}

Health and Development Foundation was tasked to develop and implement health service interventions for people who use drugs and people who are living with HIV/AIDS in the federal health care system of the Russian Federation. The federal health care system in the Russian Federation is a post-Soviet system that is publically owned and financed, with the government managing resource allocation, and where health care professionals are government employees [9]. As a centrally planned and managed system, medical service provision is free and directed from Moscow through government decrees called "prekops". The health care system is focused on the treatment of prevailing acute diseases and the prioritization of impatient treatment of acute conditions rather than chronic conditions [9]. The focus on impatient care has resulted in a vertical or "stove-piped" hospitalbased medical specialty system of separate services provided by subspecialty trained physicians [9]. Thus, when presented with the task, Health and Development Foundation noted that patients who needed Narcology services (drug treatment services) were required to seek those services at a Narcological hospital. The health services available and that are provided are specifically and strictly related to addiction treatment. Conversely, the Russian Government developed regional AIDS Centers to provide HIV/AIDS specific services for patients who are living with HIV/AIDS. The challenge for the Health and Development Foundation, was to develop specific health care interventions that would promote patients to seek and stay in care and treatment. These interventions were to be evidence-based, applicable, as well as acceptable, to both patients and providers of the health care services. Research studies have provided an evidence base for the dual epidemics of HIV/AIDS and opioid dependence which shows that integrated or colocated health care services are most effective for people who inject drugs in accessing and remaining in care and treatment [10]. Thus, Health and Development Foundation developed to the local setting, the following services and interventions for implementation to promote integrated and co-located care and for the treatment of people who inject drugs and people who are living with HIV/ AIDS.

\subsection{HIV Testing and Counseling}

Testing individuals for their HIV status is an important entry point for accessing health services for the prevention HIV and substance abuse, as well as, for care and treatment of those infected with HIV and for those who have a substance use disorder [11,12]. Testing for HIV infection, in concert with counseling for the prevention of both sexual and injection transmission of the virus, is a recognized as an evidence-based HIV prevention intervention for most-at-risk populations (MARPs), including people who inject drugs, men who have sex with men (MSM) and commercial sex workers (CSW) [5]. Health and Development Foundation developed and implemented a HIV testing and counseling curriculum along with training for the regional AIDS Centers that was MARP-focused, with a specific component on effective evidence-based counseling for injection drug users. The training component included videos of effective evidence-based counseling for injection drug users, as well as, role playing training sessions. Trainings also focused on specific aspects of counseling for the various group of clients accessing HIV testing services including: teenagers, pregnant women, MSM, CSW, and people who use drugs and their sexual partners. For health care providers in AIDS Centers, the trainings provided specific aspects of how to motivate people living with HIV/AIDS and people who inject drugs to adhere to highly active antiretroviral treatment as part of future HIV/AIDS treatment. As shown in Figure 1, upon the successful implementation of the intervention in the AIDS Centers, MARP focused HIV testing and counseling was implemented in 


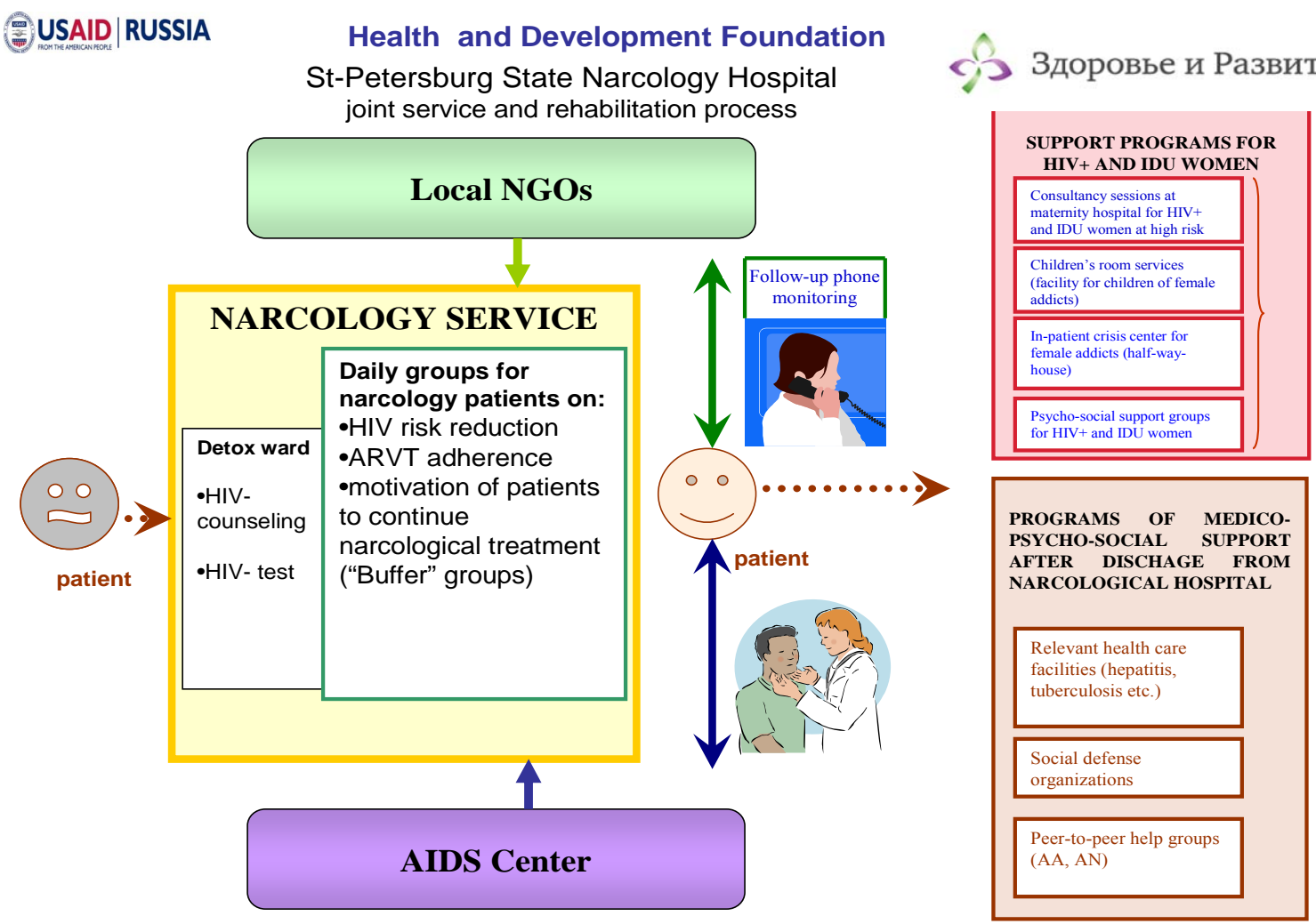

Figure 1. Health and development foundation-state narcology hospital joint service and rehabilitation process.

the Narcology detoxification treatment programs. Training for Narcologists focused on the development of specific skills in providing "patient friendly" services to people who are living with HIV/AIDS, as well as, the importance of injection drug users to adhere to highly active antiretroviral treatment. This training resulting in the initial implementation and delivery of HIV services in a drug treatment setting in the Russian Federation.

\subsection{HIVIAIDS Narcology Post-Graduate Curriculum}

The initial implementation and delivery of HIV services in Narcology hospitals and the training of Narcologists resulted in the observation that Narcologists were not knowledgeable of important HIV/AIDS medical issues related to people who inject drugs. In response, Health and Development Foundation developed, in collaboration with the Ministry of Health and Social Development, a post graduate medical curriculum entitled "The Issues of Providing Specialized Medical Care for HIV-Infected Psychoactive Substance Users". The target audience of the curriculum and trainings were medical practitioners specializing in infectious disease, including those working in specialized institutions that provide medical care to HIV-infected patients, interns with a primary specialization, physicians in a clinical residency in infectious diseases, physicians in outpatient polyclinics, physicians with higher medical education specializing in medical care, psychiatrists and narcologists. The objectives of the post-graduate training using the curriculum were to acquire and enhance an advanced knowledge and skill, both theoretical and practical, in the diagnosis, management and prevention of HIV infection in psychoactive substance users. Thus, the trainings presented the integrated approach to the medical management of both opioid dependence and HIV infection as a single chronic disease. This post graduate training was piloted in Irkutsk, Siberia and became the initial post graduate medical education course on HIV infection and substance abuse. The curriculum was subsequently approved by the Ministry of Education as the official post graduate course on HIV infection and substance abuse in the Russian Federation. An important take-home message of the educational course was the importance of co-location of HIV and drug treatment services as part of routine care and treatment of people who injection drugs. Health and Development Foundation organized collaborations between specialists of Narcology and AIDS Centers, and provided technical assistance to regional implementers to develop and integrate the care model of HIV prevention and narcology treatment. These collaborations resulted in the co-location of Narcology services, provided by narcologists, in AIDS Centers. 


\subsection{Peer Support Groups (Buffer Groups)}

Time in treatment and efficacy of the Russian Federation substance abuse detoxification and inpatient treatment programs depend substantially on the motivation of the patient to enter care and treatment and to remain in care and treatment. Unmotivated people who use drugs may enter detoxification for external reasons, such as to seek shelter or avoid family conflicts or avoid the risk of criminal prosecution. Alternatively, detoxification may be used to "refresh" oneself and lower drug use. Such individuals are most-at-risk for recidivism to opioid abuse. Developing personal motivation to address drug use and abuse, and resolve to seek recovery from drug abuse are important milestones in reducing recidivism to drug/opioid use and can be facilitated by peer support groups [13].

Health and Development Foundation developed peer or "buffer" groups as part of the federal detoxification programs as shown in Figure 1. The roles of the buffer groups were to provide a support structure for individuals undergoing detoxification, initiate a dialogue on topics related to HIV/AIDS and drug treatment, as well as, expose the patient to the approach and experience of psychological and social rehabilitation counseling. The topics addressed by the buffer groups included HIV risk reduction techniques, adherence to medications, and the development of motivation to continue in drug treatment beyond detoxification. Buffer groups worked alongside the federal medical health care providers to provide support and guidance through detoxification, as well as, to develop patient's trust in the federal health care providers and their facilities. This is a highly unique arrangement of peer drug users supporting the administration of detoxification services at the federal detoxification hospital.

\subsection{Narcological Follow-Up Phone Monitoring}

As part of the patient support structure provided by the buffer groups, Health and Development Foundation developed a further intervention where peer advocates maintained contact with individuals, post-detoxification, through cell phone contact. With patient consent, advocates obtained the cell phone number of patients upon release from detoxification and subsequently called each individual to determine their health status, as well as, each person's plan to continue in treatment for opioid dependence (see Table 1). The telephone follow-up contact provided data for the monitoring and evaluation of the interventions received and also served as a distance or remote psychological and social support intervention. The intervention component sought to increase the patient's adherence to treatment, promote risk reduction education, and inform program participants of relevant
Table 1. Patient health status determined by narcological follow-up phone monitoring after detoxification.

- Narcological Status

- Participants who did not relapse to opioid use after discharge from detoxification

- Participants who planned to continue in narcological treatment

- Participant who were in treatment after detoxification

- Retention in HIV/AIDS Services

- Participants who are aware of their last HIV test result

- Participants who are likely to under HIV Testing in the next 3 - 6 months

- Participants who attend counseling sessions at an AIDS Center after detoxification

- Participants who will seek AIDS services at an AIDS Center if their HIV test is positive

- Medication Adherence

- Participants belief in the effectiveness of antiretroviral therapy

- Participants receiving antiretroviral therapy

- Risk Reduction Behavior

○ Participants who report using sterile injection equipment the last time they injected drugs

- Participants who reported using a condom during their last sexual experience

services for people who inject drugs and people living with HIV. This follow-up, at 1, 3 and 6 months post-detoxification, is unique to the Russian health care system where it is the patient's responsibility to access and continue in the health care system without supportive interventions. As a result, currently without follow-up interventions, approximately 10 percent of patients in narcological hospitals for detoxification enter subsequent psychological and social rehabilitation in the Russian health care system.

\subsection{Women's Narcological Services}

Another gap in health services in the Russian health care system is gender-based treatment services for female drug users. To reach the female drug using population, Health and Development Foundation developed special programs focusing on females living with HIV and females who injection drugs. These included women with children, pregnant women and the families of these individuals. Providing gender-based services increased the capacity of each of the 371 pregnant women who elected to participate in the service program to have ownerships of their own health care services and identify and obtain needed social services. The medical needs of the women were complex with $36 \%$ of the women determined to be infected with HIV, 70\% infected with hepatitis $\mathrm{C}$ virus, and $71 \%$ reporting injection drug use. The goals of the women's service program were to reduce risky behavior in the context of reproductive health and substance abuse, as well as, to reduce the number of unparented (abandoned) children in Russian society. To achieve these goals the intervention comprised multiple 
components. They are: HIV and substance abuse service provision in maternity hospitals, services for children in narcological treatment programs, outpatient psychological and social support groups for women who are living with HIV/AIDS and for women who inject drugs, inpatient crisis center for women who inject drugs, and group training sessions for women who are most at risk for HIV infection and injecting drugs (see Table 2). Taken together, this component program addresses the prevention, care and treatment needs of women who are most at risk for HIV infection and drug injection, as well as, the social needs of their children. By providing a "women's centered approach" to the receipt of health care services, this program directly addresses the lack of access to health services women experience in the Russian Federation.

\subsection{Short Messaging Services for Injection Drug Users}

A recent intervention developed by the Health and Development Foundation was a short messaging service (SMS) providing short text messages related to HIV prevention, care and treatment, as well as, drug abuse prevention, care and treatment. The messages were developed by the State Narcology Hospital in St. Petersburg, Russian Federation in partnership with the Health and Development Foundation. A total of 30 messages were developed that comprised reminder messages and service information. During the 4 month program, clients

Table 2. Special programs focusing on females living with HIV/AIDS and females who injection drugs.

- HIV and substance abuse service provision in maternity hospitals

- Individual HIV prevention counseling

- Motivational counseling to enter drug treatment

- Education and information on locally available specialized HIV, narcology and reproductive health services provided by NGO's or the Federal health service

- Staff training on HIV/AIDS prevention and care services for injection drug users

- Staff training on substance abuse prevention and treatment

- Services for children in narcological treatment programs - Children's room with education specialists for children

- Outpatient psychological and social support groups for women who are living with HIV/AIDS and for women who inject drugs

- Outpatient group and individual counseling as follow-up to narcological treatment

- Counseling for women not in treatment

- Inpatient crisis center for women who inject drugs

○ "Crisis apartment" halfway house for women and their children with risk reduction counseling services, legal services, counseling for antiretroviral treatment adherence, psychological and social support groups

- Group training sessions for women who are most at risk for HIV infection and injecting drugs

- Comprehensive 5 day counseling with subspecialty sessions including teacher, psychotherapist, psychologist, gynecologist, lawyer, peer HIV consultant registered their cell phone number with the hospital and received one or two messages per week. The participant could unsubscribe at any time. While short messaging services have been recently developed for HIV/AIDS targeting specific populations, this is the first known short messaging service directed to people who inject drugs $[14,15]$.

\section{EVALUTATION AND DISCUSSION}

The model program for retention and continuity of care and treatment for opioid dependent injection drug users was extensively evaluated using both personal indictors to assure consistent demographics among the groups and program outcome indicators to analyze the outcomes for each intervention. As shown in Figure 1, the interventions developed by Health and Development Foundation occurred longitudinally as the patient/client proceeded through the Federal Narcological Detoxification Service onto and through the rehabilitation services. As the patient/client entered the detoxification services the initial intervention encountered is the integrated HIV testing and counseling. As detoxification progressed, the client encountered the buffer groups that provided support and counseling. Subsequent to detoxification, follow-up occurred via telephone monitoring with case management services directing the female clients to genderbased rehabilitation and social services. Over the course of the rehabilitation services, the patient/client could subscribe to the short message service for people who inject drugs. Of the 1618 recruited to participate in the model program, $79.6 \%$ agreed to the targeted interventions indicating that the model interventions were well received and accepted by people who inject drugs. Retention in the project over the 6 month monitoring period was $83 \%$, both at one and three months, and $72 \%$ at six months. The provision of ancillary and support services, such as case management, housing, food, transportation, mental health and substance abuse treatment are used by the HIV medical system and NGO's as facilitative strategies directed to populations that have difficulty entering or staying in HIV primary car, such as people who inject drugs [16]. Thus, for this model program approximately 3 out of 4 program entrants remained in care after six months. Remaining in care had a significant effect on health status with $54 \%$ of those in care in remission for drug use; while only $23 \%$ of those out of care where in remission $(\mathrm{p}=0.05$, Student's $\mathrm{T}$ Test).

Peer mentoring has been shown to be an important intervention to reduce HIV transmission and promote access to care and treatment [17]. For those accepting buffer group support and counseling and follow-up telephone monitoring, $57 \%$ accessed narcological treatment after detoxification, while only $45 \%$ of those not receiv- 
ing peer support and monitoring during detoxification entered further narcological treatment $(p=0.06)$. In addition, greater than $96 \%$ of buffer group/monitoring participants compared to $23 \%$ of nonparticipants indicted a likelihood of taking a HIV test in the next $3-6$ months. $(p=0.03)$. Accessing and remaining in substance abuse treatment and willingness to take an HIV test are important predictors in reducing HIV transmission and with HIV medication adherence in people who inject drugs $[17,18]$. Interestingly, there was no difference between the groups regarding the opinion of the effectiveness of antiretroviral treatment for HIV infection, with $88 \%$ and $90 \%$ expressing the same opinion regarding treatment effectiveness. However, the buffer group/monitoring group reported a higher degree of medication adherence to antiretroviral treatment $(68 \%$ to $43 \%)$ when asked if they had taken their daily medication one month after detoxification $(p=0.04)$. These data indicate the importance of peer-led interventions and follow-up phone monitoring for improved HIV prevention and treatment behaviors for people who inject drugs.

Three month evaluation data are available for the short messaging services for people who inject drugs with $79 \%$ of participants expressing satisfaction with the HIV content of the messaging, 54\% satisfied with the substance abuse treatment content, $47 \%$ satisfied with the psychosocial support content and $29 \%$ satisfied with the gender-based content. The majority of message recipients expressed a need to see more content for each category. Approximately three of four message recipients kept the message content for use later, while approximately $40 \%$ of recipients called hotlines provided in the messages or shared message content with others. Roughly one in three recipients entered internet sites provided in the messages. Thus, people who inject drugs were overall satisfied with the short messaging service and managed the message content on receipt. These preliminary data suggest that text messages to mobile phones of people who inject drugs can be an effective tool to provide health care (HIV and substance abuse treatment) information that will be processed and acted upon by the recipient.

\section{CONCLUSION}

The model program and interventions taken as a whole, HIV Testing and Counseling, HIV/AIDS Narcology Post-Graduate Curriculum, Peer Support Groups (Buffer groups), Narcological Follow-up Phone Monitoring, Women's Narcological Services and Short Messaging Services for Injection Drug Users were developed and implemented into the Russian Federal Detoxification System for people who inject drugs and are opioid dependent. As such, with staff training, the interventions were accepted by the federal medical care providers and shown to be effective in enhancing access to care and treatment as well as retention in care and treatment. These interventions provide a model for scale-up that will enhance the health care service environment for people who inject drugs and their perception of the federalbased drug treatment system in the Russian Federation.

\section{ACKNOWLEDGEMENTS}

The authors acknowledge the significant collaboration of the Ministry of Health and Social Development, Russian Federation and the Federal Narcological Services of the Russian Federation.

\section{REFERENCES}

[1] Kresina, T.F., Bruce, R.D., Pirard, S., Mulvey, K., Huesca, R.S. and Lubran, R. (2012) International expansion of the use of pharmacotherapies for the treatment of opioid dependence. Clinical and Experimental Pharmacology, in press.

[2] United Nations Office of Drugs Control (UNODC) (2010) World Drug Report 2010. United Nations Publications, New York, 42-51.

http://www.unodc.org/unodc/en/data-and-analysis/WDR2010.html

[3] World Health Organization (2010) The international statistical classification of diseases and related health problems. 10th Edition, WHO Press.

http://apps.who.int/classifications/icd10/browse/2010/en

[4] World Health Organization (2009) Guidelines for the psychosocially assisted pharmacological treatment of opioid dependence. WHO Press.

http://www.who.int/substance_abuse/publications/opioid dependence guidelines.pdf

[5] World Health Organization (2010) Priority interventions. HIV/AIDS prevention, treatment and care in the health sector. World Health Organization HIV/AIDS Department. http://www.who.int/hiv/pub/guidelines/9789241500234/e n/index.html

[6] Kirzhanova, V.V. and Sidoryuk, O.V. (2010) Indicators of general and primary morbidity-related drug disorders in the Russian Federation during 1999-2009. http://www.nrca-rf.ru/2 195.html

[7] Information bulletin No. 34 of the federal scientificmethodological center for the prevention and control of AIDS in the Russian Federation (2010). http://hivrussia.org/files/bul34.pdf

[8] Information bulletin No. 33 of the federal scientificmethodological center for the prevention and control of AIDS in the Russian Federation (2009). http://hivrussia.org/files/bul_33.pdf

[9] Antoun, J., Phillips, F. and Johnson, T. (2011) Post-soviet transition: Improving health services delivery and management. Mount Sinai Journal of Medicine, 78, 436-448. doi:10.1002/msj.20261

[10] Kresina, T.F., Lubran, R. and Cheever, L.W. (2011) Substance abuse treatment utilizing medication assisted treatment as HIV prevention. In: HIV Prevention, Intech Pub- 
lishers, Rijeka, 527-548.

[11] Lawn, S.D., Fraenzel, A., Kranzer, K., Caldwell, J., Bekker, L.G. and Wood, R. (2011) Provider-initiated HIV testing increases access of patients with HIV-associated tuberculosis to antiretroviral treatment. South African Medical Journal, 101, 258-262.

[12] Volkow, N.D. and Montaner, J. (2011) The urgency of providing comprehensive and integrated treatment for substance abusers with HIV. Health Affairs (Millwood), 30, 1411-1419. doi:10.1377/hlthaff.2011.0663

[13] Tracy, K., Burton, M., Nich, C. and Rounsaville, B. (2011) Utilizing peer mentorship to engage high recidivism substance-abusing patients in treatment. American Journal of Drug and Alcohol Abuse, 37, 525-531. doi:10.3109/00952990.2011.600385

[14] Mukund Bahadur, K.-C. and Murray, P.J. (2010) Cell phone short messaging service (SMS) for HIV/AIDS in South Africa: A literature review. Studies in Health Technology and Informatics, 160, 530-534.

[15] Wright, E., Fortune, T., Juzang, I. and Bull, S. (2011) Text messaging for HIV prevention with young black men: Formative research and campaign development. AIDS Care, 23, 534-541. doi:10.1080/09540121.2010.524190

[16] Conviser, R. and Pounds, M.B. (2002) Background for the studies on ancillary services and primary care use. AIDS Care, 14, S7-S14. doi:10.1080/09540120220149993

[17] Purcell, D.W., Latka, M.H., Metsch, L.R. and INSPIRE Study Team (2007) Results from a randomized controlled trial of a peer-mentoring intervention to reduce HIV transmission and increase access to care and adherence to HIV medications among HIV-seropositive injection drug users. Journal of the Acquired Immune Deficiency Syndrome, 46, S35-S47.

[18] Roux, P., Carrieri, M.P., Cohen, J., Ravaux, I., PoizotMartin, I., Dellamonica, P. and Spire, B. (2009) Retention in opioid substitution treatment: A major predictor of long-term virological success for HIV-infected injection drug users receiving antiretroviral treatment. Clinical Infectious Diseases, 49, 1433-1440. doi:10.1086/630209 